\title{
Partial activity is seen with many substitutions of highly conserved active site residues in human Pseudouridine synthase 1
}

\author{
BRYAN S. SIBERT, ${ }^{1}$ NATHAN FISCHEL-GHODSIAN, ${ }^{2}$ and JEFFREY R. PATTON ${ }^{\mathbf{1}}$ \\ ${ }^{1}$ Department of Pathology, Microbiology, and Immunology, University of South Carolina, School of Medicine, Columbia, South Carolina 29208, USA \\ ${ }^{2}$ Cedars-Sinai Medical Center, Medical Genetics Institute, Ahmanson Department of Pediatrics, Steven Spielberg Pediatric Research Center, \\ David Geffen School of Medicine, University of California at Los Angeles, Los Angeles, California 90048, USA
}

\begin{abstract}
Pseudouridine synthase 1 (Pus1p) is an enzyme that converts uridine to Pseudouridine ( $\Psi$ ) in tRNA and other RNAs in eukaryotes. The active site of Pus1p is composed of stretches of amino acids that are highly conserved and it is hypothesized that mutation of select residues would impair the enzyme's ability to catalyze the formation of $\Psi$. However, most mutagenesis studies have been confined to substitution of the catalytic aspartate, which invariably results in an inactive enzyme in all $\Psi$ synthases tested. To determine the requirements for particular amino acids at certain absolutely conserved positions in Pus1p, three residues (R116, Y173, R267) that correspond to amino acids known to compose the active site of TruA, a bacterial $\Psi$ synthase that is homologous to Pus1p, were mutated in human Pus1p (hPus1p). The effects of those mutations were determined with three different in vitro assays of pseudouridylation and several tRNA substrates. Surprisingly, it was found that each of these components of the hPus1p active site could tolerate certain amino acid substitutions and in fact most mutants exhibited some activity. The most active mutants retained near wild-type activity at positions 27 or 28 in the substrate tRNA, but activity was greatly reduced or absent at other positions in tRNA readily modified by wild-type hPus1p.
\end{abstract}

Keywords: pseudouridine; synthase; tRNA; modification; mutagenesis

\section{INTRODUCTION}

Pseudouridine $(\Psi)$ is a post-transcriptionally modified form of uridine found in stable RNAs such as ribosomal RNA (rRNA), transfer RNA (tRNA), and small nuclear RNA (snRNA) (Bjork et al. 1987; Massenet et al. 1998; Ofengand 2002). It is one of the most abundant modified nucleotides and the positions of $\Psi$ residues within RNAs are well conserved (Auffinger and Westhof 1998). Pseudouridine plays an important role in the stabilization of the secondary and tertiary structure of tRNA and studies have indicated that it stabilizes RNA base stacking and codon-anticodon interactions (Davis and Poulter 1991; Davis 1995; Davis et al. 1998). In addition, $\Psi$ modifications in snRNAs have been shown to be necessary for pre-mRNA splicing in a Xenopus oocyte splicing system (Yu et al. 1998; Zhao and Yu 2004)

Reprint requests to: Jeffrey R. Patton, Department of Pathology, Microbiology, and Immunology, University of South Carolina, School of Medicine, Columbia, SC 29208, USA; e-mail: patton@med.sc.edu; fax: (803) 733-3192.

Article published online ahead of print. Article and publication date are at http://www.rnajournal.org/cgi/doi/10.1261/rna.984508. and in an in vitro reconstitution/complementation assay using HeLa cell extracts (Donmez et al. 2004). The presence of $\Psi$ has also been shown to play a critical role in the regulation of transcription by nuclear receptors (Zhao et al. 2004b, 2007).

This modification is formed by a group of enzymes known as pseudouridine synthases, of which there are five families: RluA, RsuA, TruA, TruB, and TruD (Hamma and Ferré-D’Amaré 2006). While there is significant homology among $\Psi$ synthases in a family, homology between the various families is relatively low (Koonin 1996; Hamma and Ferré-D’Amaré 2006). Of the eukaryotic $\Psi$ synthases, the TruA family has been studied most extensively, and human Pseudouridine synthase 1 (hPuslp) is a member of this family. Saccharomyces cerevisiae Pus1p was shown to modify uridines at 1,26, 27, 28, 34, and 36 in vitro, and 65 and 67 in vivo, as well as position 43 in U2 snRNA in vitro and in vivo (Motorin et al. 1998; Massenet et al. 1999). Mouse Puslp (mPuslp) has been shown to modify positions 27, 28, 34, and 36 in vitro (Chen and Patton 1999). Additionally, positions 1 and 30 on tRNA and position 43 on S. cerevisiae U2 snRNA are modified when mPuslp was 
expressed in a Puslp-deficient S. cerevisiae strain (BehmAnsmant et al. 2006). When the pus1 gene was knocked out in Caenorhabditis elegans there was no effect on the modification of U2 snRNA isolated from worms, but the modification of tRNAs at Pus1p-dependent positions 27 and 28 was lost (Patton and Padgett 2003).

From the crystal structure of bacterial TruA it is known that the active site of this original member of the TruA family is populated by four strictly conserved amino acids (Foster et al. 2000). These include a catalytic aspartate (D60 in TruA), two arginines (R58 and R205), and a tyrosine (Y118). These residues correspond to D118, R116, R267, and Y173 in hPuslp (see Fig. 1). In addition to the conservation of these four amino acids among the TruA $\Psi$ synthases, there is a particularly high level of sequence homology in the areas immediately surrounding these residues (see Fig. 1). Previous mutation studies on $\Psi$ synthases have shown that the catalytic aspartate is absolutely critical for the enzymatic activity and cannot be replaced with any other amino acid (Huang et al. 1998; Conrad et al. 1999; Ramamurthy et al. 1999; Raychaudhuri et al. 1999; Zebarjadian et al. 1999; Del Campo et al. 2001; Kaya and Ofengand 2003; Zhao et al. 2004b). Analysis of the TruA crystal structure in addition to structural studies of members of other $\Psi$ families has suggested that the two arginines (R58 and R205 in TruA) play a role in constraining the aspartate and in flipping out the uracil base of the substrate allowing it to be modified (Foster et al. 2000; Hamma and Ferré-D'Amaré 2006; Hur et al. 2006; Hur and Stroud 2007). The role of the tyrosine at 118 in TruA has not been fully elucidated; however, a previous mutation study using TruB $\Psi$ synthase has shown that replacement of the tyrosine with alanine, phenylalanine, or leucine results in the lack of enzymatic activity on a natural substrate (Phannachet et al. 2005).

In humans, a single nucleotide mutation in the PUS1 gene results in the change of the arginine at 116 to tryptophan and has been correlated with the disease mitochondrial myopathy and sideroblastic anemia (MLASA) (Bykhovskaya et al. 2004). Extracts from patient derived cell lines show no hPus1p activity in vitro, and tRNAs from those cell lines lack $\Psi$ at positions in

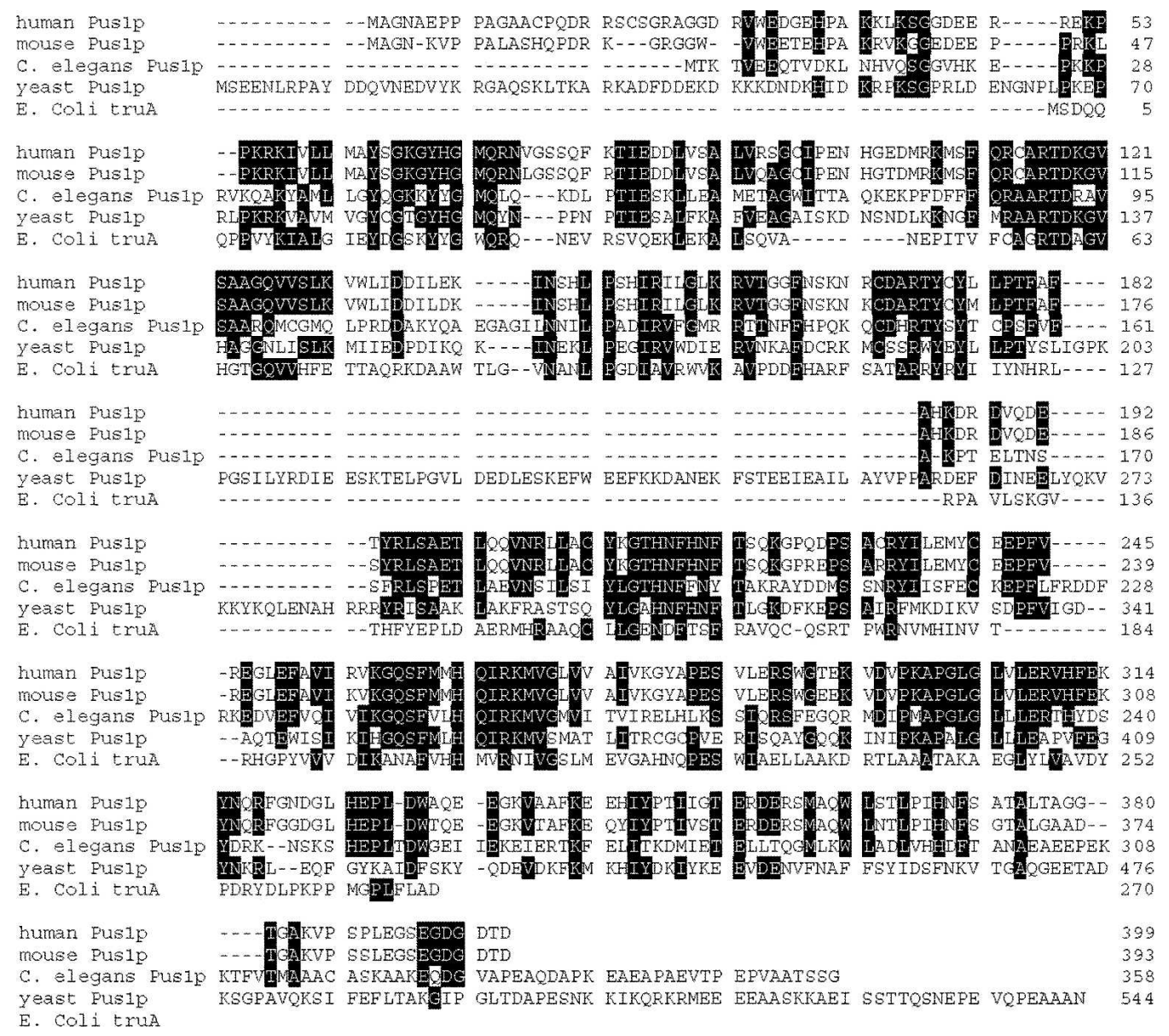

FIGURE 1. The amino acid sequences of human, mouse, C. elegans, and S. cerevisiae Puslp, was well as E. coli TruA are aligned to show homology. Positions where at least three of the five proteins show identity have been highlighted. 
tRNAs expected to be modified by the hPuslp enzyme, even though the mutant protein is still present (Patton et al. 2005). Since this is not the position of the critical catalytic aspartate (D118) it has been suggested that the nonconservative replacement found in the patients, $\mathrm{R} 116 \mathrm{~W}$, sterically hinders the active site and results in an inactive enzyme.

We hypothesized that any substitution at these highly conserved sites in hPus1p $(116,173$, and 267) would affect the function of the synthase and that anything other than conservative replacement would not be tolerated. To test this we created a number of single amino acid replacement mutants at these positions using both conservative and nonconservative substitution. Surprisingly, many of the mutants retained partial $\Psi$ synthase activity at positions in tRNA known to be modified by Puslp, and these mutants exhibit differential activities at particular positions on tRNA substrates instead of exhibiting a general reduction of activity.

\section{RESULTS}

The wild-type hPuslp used in these studies (see Fig. 1) is predicted from the DNA sequence to have an additional 51 amino acids on the amino terminus compared with the partial human sequence published previously (Chen and Patton 1999). A partial human protein, starting with the methionine at position 62 , which is in the middle of a region conserved in TruA synthases (see Fig. 1), was expressed in bacteria and the isolated protein had no activity (Chen and Patton 1999). The wild-type hPuslp used in this study, with the additional amino acids, starting with position 1 in Figure 1, containing a histidine leader at the amino terminus, was expressed in bacteria, isolated, and tested in $\Psi$ synthase assays (see below).

In order to elucidate the role of active site amino acids R116, R267, and Y173 in hPuslp, we used site-directed mutagenesis to create mutations at these positions. The mutations included both conservative and nonconservative replacements at each site. Characteristics of Puslp include its specificity for the same positions on a wide variety of tRNAs and its multisite specificity (Motorin et al. 1998; Chen and Patton 1999; BehmAnsmant et al. 2006). For this reason, we chose to test the mutants on multiple tRNA substrates. All of the substrates were generated using in vitro transcrip- tion as described in the methods, and the sequences of all of the substrates used can be found in Figure 2.

The assay used as a first screen of wild-type and mutant activities is the tritium release assay, which provides a quantitative measure of total $\Psi$ formation following incubation of the enzyme with uridine $5-{ }^{3} \mathrm{H}$-labeled substrate. It is a simple assay that allows for the testing of the numerous mutant enzymes and several tRNA substrates in order to quantitate total activity (Table 1 ). The values in the table indicate the number of moles $\Psi$ produced per mole of tRNA. One mole $\Psi /$ mole tRNA is expected for each predicted position; however, some positions may not be completely modified in vitro, resulting in a lower than expected number even for wild-type hPus1p. The number of possible positions for each substrate can be found at the top of the columns, and most have already been shown to be modified by mPuslp in vitro (Chen and Patton 1999; BehmAnsmant et al. 2006). Since the human enzyme is highly homologous to mPus1p (90\% identity, $4 \%$ conservative replacement) (see Fig. 1), it was expected hPus1p would also modify all the same substrates at the same positions.

Since mPuslp is well characterized (Chen and Patton 1999, 2000; Behm-Ansmant et al. 2006) and this is the first test of hPuslp in vitro, the activities for mPuslp and hPuslp were compared. The two $\Psi$ synthases had very similar activities

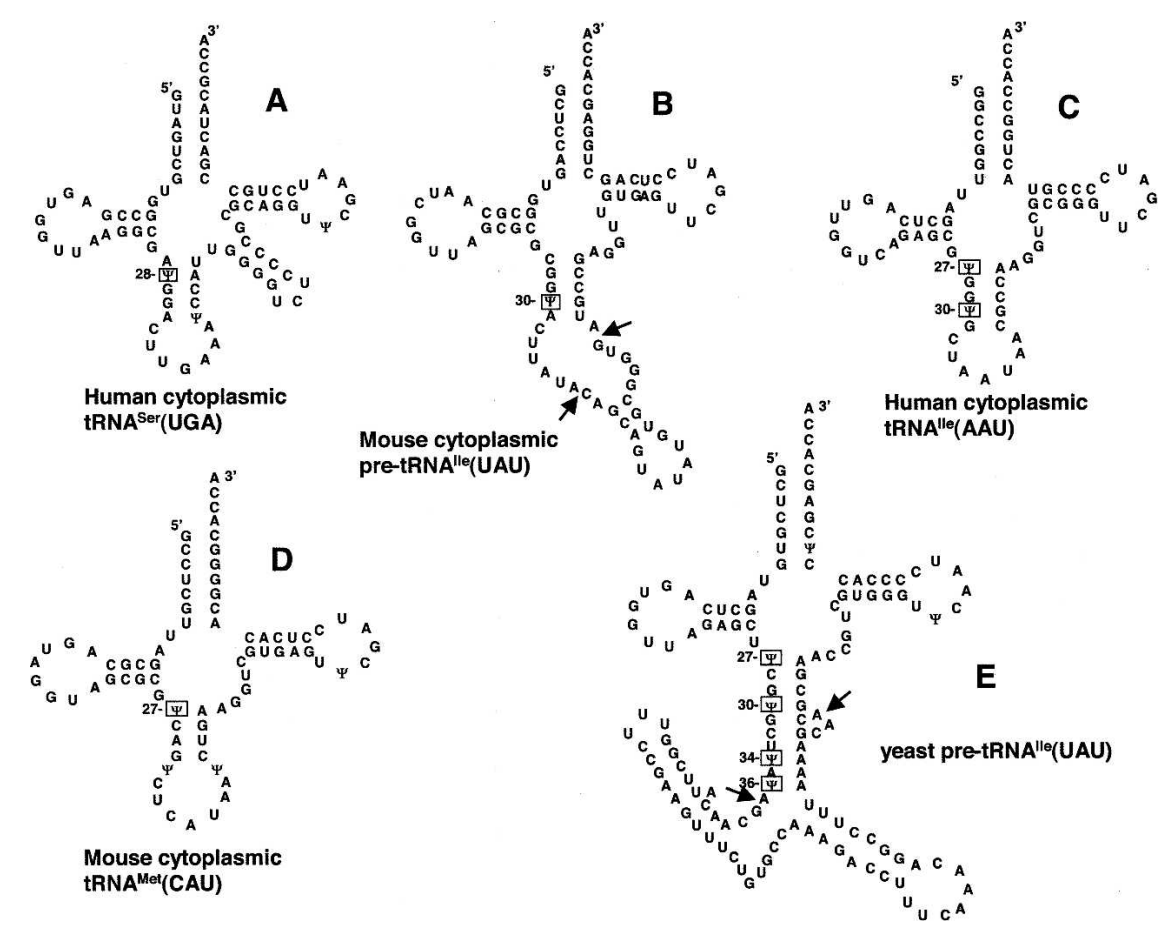

FIGURE 2. Structures of tRNA substrates used to test the activity of wild-type and mutant hPuslps. The $\Psi$ residues that are modified by hPuslp are surrounded by a rectangle and the position denoted to the left. Human cytoplasmic tRNA $\mathrm{UGA}_{\mathrm{Se}}^{\mathrm{Ser}}(A)$, mouse cytoplasmic pretRNA $A_{\mathrm{UAU}}^{\mathrm{Ile}}(B)$, human cytoplasmic tRNA $\mathrm{AAU}_{\mathrm{AI}}^{\mathrm{Ile}}(C)$, and mouse cytoplasmic tRNA Met $(D)$ are shown with predicted secondary structures. In the $S$. cerevisiae pre-tRNA ${ }^{\text {Ile }}(B, E)$, the arrows define the intron of the pre-tRNA. 
TABLE 1. ${ }^{3} \mathrm{H}$-release assays of mPus $1 \mathrm{p}, \mathrm{hPus} 1 \mathrm{p}$, and position 116 mutants with various substrates

\begin{tabular}{lcccc}
\hline Enzyme & $\begin{array}{c}\text { S. cerevisiae pre-tRNA } \\
(\Psi \text { at } 27,30,34,36)^{\mathrm{a}}\end{array}$ & $\begin{array}{c}\text { Mouse } \\
\text { RNA }^{\text {Met }} \\
(\Psi \text { at } 27)^{\mathrm{b}}\end{array}$ & $\begin{array}{c}\text { Mouse } \\
\text { pre-tRNA } \\
(\Psi \text { at 30) }\end{array}$ & $\begin{array}{c}\text { Human } \\
\text { tRNA } \\
(\Psi \text { at 27, 30 })\end{array}$ \\
\hline mPus1p & $3.12 \pm 0.67(n=2)$ & 0.91 & N.D. & N.D. \\
hPus1p & $3.12 \pm 0.63(n=4)$ & 0.98 & 0.77 & $0.92 \pm 0.03(n=2)$ \\
R116A & $0.46 \pm 0.25(n=2)$ & 0.09 & $<0.01$ & 0.74 \\
R116C & $1.18 \pm 0.03(n=2)$ & N.D. & N.D. & $0.74 \pm 0.04(n=2)$ \\
R116E & 0.00 & N.D. & N.D. & N.D. \\
R116G & 0.44 & N.D. & $<0.01$ & 0.67 \\
R116H & $0.06 \pm 0.04(n=2)$ & 0.01 & N.D. & N.D. \\
R116K & $1.33 \pm 0.15(n=3)$ & 0.76 & 0.02 & $0.80 \pm 0.01(n=2)$ \\
R116N & 0.46 & N.D. & 0.01 & 0.72 \\
R116Q & 0.20 & N.D. & N.D. & N.D. \\
R116S & 0.59 & N.D. & 0.01 & 0.73 \\
R116W & $0.03 \pm 0.02(n=3)$ & 0.01 & N.D. & N.D. \\
\hline A616ity & & & &
\end{tabular}

Activity is reported in mol $\Psi / \mathrm{mol}$ tRNA.

${ }^{a}$ The positions of $\Psi$ that are possible Pus $1 \mathrm{p}$ modification sites are given under the substrate in the column heading. The activity is in mol $\Psi / \mathrm{mol}$ tRNA and has had the value measured for incubation of the substrate for the same length of time with bacterially expressed Lac subtracted. A value of " $0.00^{\prime \prime}$ means there was no activity above background in this sample. With yeast pre-tRNA ${ }^{\text {lle }}$ and human tRNA $A_{A A U}^{l l e}, n$ denotes the number of determinations on separate days and, when $n$ is not given, it is equal to 1 . With mouse tRNA ${ }^{\text {Met }}$ and pretRNA ${ }_{\cup A U}^{\text {lle }}$ the values reported are the means of three assays of the same reaction and all of the SDs were $<0.03$ for $t R N A^{\text {Met }}$ and 0.01 for mouse pre-tRNA ${ }_{\cup A U}^{\text {lle }}$

${ }^{\mathrm{b}} \mathrm{N}$.D. denotes "not determined."

the $\mathrm{R} 116 \mathrm{H}$ mutation, it is much more constrained than the side chain for arginine.

With the $m$ tRNA ${ }^{\text {Met }}$ substrate, where only one position (27) (see Fig. 2) is expected to be modified by hPus1p, the results are very interesting. The wildtype mPuslp and hPuslp show the formation of nearly $1 \mathrm{~mol} \Psi / \mathrm{mol}$ tRNA as reported above, but the $\mathrm{R} 116 \mathrm{~K}$ mutant exhibited an activity of 0.76 mol $\Psi /$ mol tRNA for this substrate, a level of activity that approaches that of wild type. The other mutants tested with the $m$ tRNA ${ }^{\text {Met }}$ substrate had either low levels of activity, as with $\mathrm{R} 116 \mathrm{~A}$, or no activity, as with $\mathrm{R} 116 \mathrm{H}$ and R116W.

All of the mutants that showed some activity with the y pre-tRNA ${ }^{\text {Ile }}$ substrate were tested on human tRNA $A_{A A U}^{\text {Ile }}$ (h tRNA $A_{A A U}^{\text {Ile }}$ ). This tRNA has two possible uridines that could be modified by hPus1p, positions 27 and 30 . Wild-type hPus1p only shows activity at the level of $0.92 \mathrm{~mol} \Psi / \mathrm{mol}$ tRNA (Table 1 ).

(Table 1) on S. cerevisiae pre-tRNA ${ }^{\text {Ile }}$ (y pre-tRNA ${ }^{\text {Ile }}$ ) and mouse tRNA ${ }^{\text {Met }}\left(\mathrm{m}\right.$ tRNA $\left.{ }^{\text {Met }}\right)$. Each modified the y pretRNA $^{\text {Ile }}$ at slightly higher than $3 \mathrm{~mol}$ of $\Psi / \mathrm{mol}$ tRNA, denoting that at least three uridines have been converted to $\Psi$. With the $m$ tRNA ${ }^{\text {Met }}$ substrate, again the activities were quite similar, with $\sim 1 \mathrm{~mol} \Psi / \mathrm{mol}$ tRNA for each of the two enzymes, the expected level of $\Psi$ with this substrate.

Next we tested hPuslp mutants at the R116 position, the residue that is mutated to tryptophan in some patients with MLASA (Bykhovskaya et al. 2004). The mutants can be divided into three groups based upon their activity with $y$ pre-tRNA ${ }^{\text {Ile }}$. R116K and R116C retained the most activity with approximately one-third that of wild-type levels. The replacement of arginine with lysine is a conservative one, retaining the charge and approximate size of the arginine side chain. The side chain on cysteine is not charged at neutral $\mathrm{pH}$ and its size is considerably smaller than that of arginine or lysine.

The second group, composed of the R116A, R116G, R116N, R116Q, and R116S mutants, all exhibited some activity with the y pre-tRNA ${ }^{\text {Ile }}$ substrate (Table 1). All of the side chains for these amino acids are relatively small and are either nonpolar or polar and uncharged at neutral $\mathrm{pH}$. The R116E, R116H, and R116W (the mutation in MLASA patients) mutants make up the third group and were essentially without activity. Tryptophan has a large bulky side chain and glutamic acid's side chain is negatively charged, replacing the positively charged side chain of arginine. Although the positive charge is retained with
This suggests that approximately one site is being modified by hPus1p, most likely position 27 . Nonetheless, all the mutants that were tested had significant activity (between 0.67 and $0.80 \mathrm{~mol} \Psi / \mathrm{mol}$ tRNA) on this substrate, a result reminiscent of the activity of R116K on $\mathrm{m} \mathrm{tRNA}^{\mathrm{Met}}$; but with $h$ tRNA $\mathrm{Ale}$, even R116A, C, G, N, and S have significant levels of activity, compared with wild-type hPus1p.

When mouse pre-tRNA $\mathrm{IAUU}_{\mathrm{UAU}}^{\mathrm{lle}}\left(\mathrm{m}\right.$ pre-tRNA $\mathrm{UAU}_{\mathrm{UAU}}^{\mathrm{Ile}}$ ) (see Fig. 1) is used as a substrate, where only position 30 can be modified by hPus1p, only the wild-type enzyme exhibits any appreciable activity. There is a clear dichotomy between wild-type hPuslp and all the mutants tested, with wild-type hPus1p exhibiting $0.77 \mathrm{~mol} \Psi / \mathrm{mol}$ tRNA, and $\mathrm{R} 116 \mathrm{~K}$, the mutant with the highest activity, is limited to $0.02 \mathrm{~mol} \Psi / \mathrm{mol}$ tRNA.

Tritium release assays were also performed with mutants at the $\mathrm{Y} 173$ and R267 positions to determine if any activity would be retained when these strictly conserved amino acids were replaced. Table 2 contains the tritium release data for the Y173 and R267 mutants incubated with the y pre-tRNA ${ }^{\text {Ile }}$ substrate, conducted in separate experiments from those presented in Table 1. The Y173C, Y173F, Y173G, and Y173T mutants all retained some activity; however, it was relatively low $(0.54 \mathrm{~mol} \Psi / \mathrm{mol}$ tRNA at best) (Table 2). The combination of hydroxyl group and hydrophobic ring appears to be required for activity since Y173F, Y173T, and Y173S are minimally active or totally inactive. 
TABLE 2. ${ }^{3} \mathrm{H}$-release assays of hPus $1 \mathrm{p}$ and position 173 and 267 mutants with $S$. cerevisiae pre-tRNA $\cup$ lle

\begin{tabular}{lc}
\hline Enzyme & $\begin{array}{c}\text { S. cerevisiae pre-tRNA } \\
(\Psi \text { at } 27,30,34,36)^{\mathrm{a}}\end{array}$ \\
\hline hPus1p & $2.94 \pm 0.48 .(n=3)$ \\
Y173C & $0.52 \pm 0.06 .(n=2)$ \\
Y173F & $0.25 \pm 0.08 .(n=2)$ \\
Y173G & $0.29 \pm 0.04 .(n=2)$ \\
Y173S & $0.01 \pm 0.00 .(n=2)$ \\
Y173T & $0.54 \pm 0.07 .(n=2)$ \\
R267G & 0.09 \\
R267K & 1.12
\end{tabular}

Activity is reported in mol $\Psi / \mathrm{mol}$ tRNA.

${ }^{a}$ The positions of $\Psi$ that are possible Pus1p modification sites are given under the substrate in the column heading. The activity in $\mathrm{mol} \Psi / \mathrm{mol}$ tRNA has had the value measured for incubation of the substrate for the same length of time with bacterially expressed Lac subtracted. The number of determinations on separate days used to obtain the mean is stated in parentheses. With the R267 mutants the values reported are the means of three assays of the same reaction and the SDs for R267G and R267K are $<0.01$ and 0.03, respectively.

From the structure of TruA, the arginines at 116 and 267 might be considered equivalent positions and the R267K mutant retained approximately one-third of wild-type activity with the y pre-tRNA ${ }^{\text {Ile }}$ substrate (Table 2), the same level observed with R116K. The same correspondence is not true for the R267G mutant, which exhibited negligible activity with this substrate $(0.09 \mathrm{~mol} \Psi / \mathrm{mol}$ tRNA) (Table 2), considerably less than that of R116G (0.44 mol $\Psi / \mathrm{mol}$ tRNA) (Table 1).

Since the data in Table 1 suggested that certain mutants might modify position 27 , the R116K and R267K mutants were chosen for further analysis employing substrates that can be modified by hPus1p at only one residue, position 27 $\left(\mathrm{m}\right.$ tRNA $^{\mathrm{Met}}$ ) or position 28 (h tRNA ${ }^{\mathrm{Ser}}$ ). Wild-type hPuslp and the R116K mutant were incubated with $\mathrm{m}$ tRNA $^{\text {Met }}$, time points were taken at 15, 30, 60, and $120 \mathrm{~min}$, and the levels of activity were measured using the tritium release assay. Wild-type hPus1p and the R267K mutant were incubated with $\mathrm{h}$ tRNA ${ }^{\text {Ser }}$ for 30,60 , and $120 \mathrm{~min}$. As can be seen in Figure 3A, the R116K mutant showed a near wild-type rate of $\Psi$ formation and final level of pseudouridylation. The $\mathrm{R} 267 \mathrm{~K}$ rate and total activity on $\mathrm{h}$ tRNA ${ }^{\text {Ser }}$ was indistinguishable from that of wild-type hPuslp (Fig. $3 \mathrm{~B})$. This indicates that, at least for positions 27 and 28 in long-term incubations, the activity of these mutant enzymes is nearly equal to that of wild-type hPus1p.

However, short-term kinetic analysis (1- and 2-min incubations) (see Materials and Methods) of wild-type hPuslp and the R116K mutant using the y pre-tRNA ${ }^{\text {Ile }}$ substrate yielded apparent $K_{m}$ values of $32( \pm 1) \mathrm{nM}$ for wild-type hPus1p and $168( \pm 20) \mathrm{nM}$ for the R116K mutant. The $V_{\max }$ values determined were $54( \pm 4)$ and $56( \pm 8) \mathrm{fmol} / \mathrm{min}$ for wild-type and the R116K mutant, respectively. The equilibrium binding constants obtained with the same substrate are $250 \mathrm{nM}$ for wild-type hPuslp and $420 \mathrm{nM}$ for R116K.

In order to determine whether the mutant enzymes were preferentially modifying certain positions on tRNA substrates that have more than one position that can be modified, or if all of the possible positions were being incompletely modified, an assay other than ${ }^{3} \mathrm{H}$-release was required. The CMCT $\Psi$-mapping assay, where there is a reverse transcription stop one position before a covalently modified $\Psi$, was used to precisely determine which uridine was modified on the y pre-tRNA ${ }^{\text {Ile }}$ substrate after incubation with R116C, R267K, and wild-type hPus1p.

The presence of $\Psi$ is indicated by more intense bands in the CMCT lanes than in the control lane $(-\mathrm{CMCT})$ one position prior to a U. Arrows in Figure 4 denote the positions beneath the predicted $\Psi$ s where the stops should be observed. In Figure 4A we show that wild-type hPuslp creates $\Psi$ at all predicted positions: 27, 30, 34, and 36 (Fig. $4 \mathrm{~A}$, lanes 1-3). The level of $\Psi$ at position 36, even with wild-type hPuslp, is considerably less than that of the other positions (27, 30, and 34), which agrees with earlier characterization of mPus1p (Behm-Ansmant et al. 2006). The CMCT result is consistent with the tritium release assay data where a value of $\sim 3.00 \mathrm{~mol} \Psi / \mathrm{mol}$ tRNA was

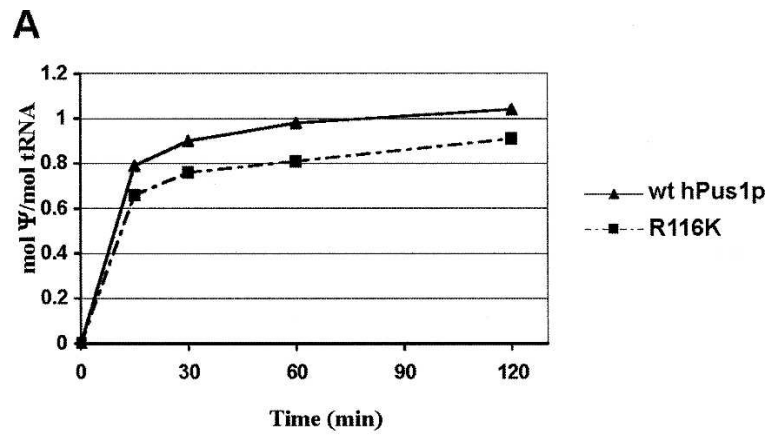

B

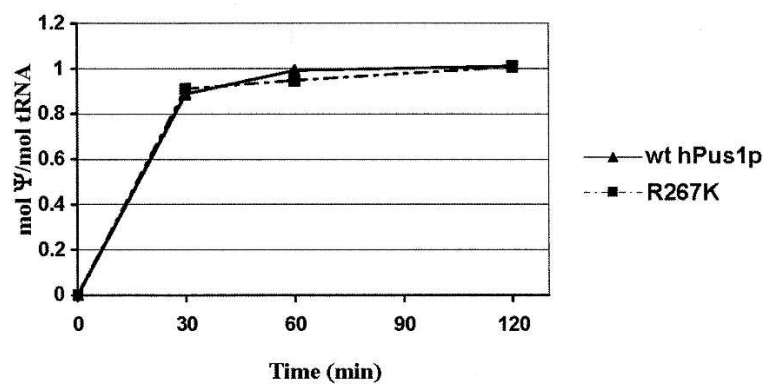

FIGURE 3. Time course of wild-type and mutant hPuslp activity. Reactions were incubated for the times shown and the levels of $\Psi$ formed were determined by ${ }^{3} \mathrm{H}$-release assays (see Materials and Methods). For the $\mathrm{m}$ tRNA ${ }^{\text {Met }}$ substrate ( $A$, wt hPuslp and the R116K mutant) the SDs for the three assays of the same reaction were all $\leq 0.04$ mol $\Psi /$ mol tRNA. With the h tRNASer substrate ( $B$, wt hPuslp and the R267K mutant) only a single assay was carried out for each time point. 
A

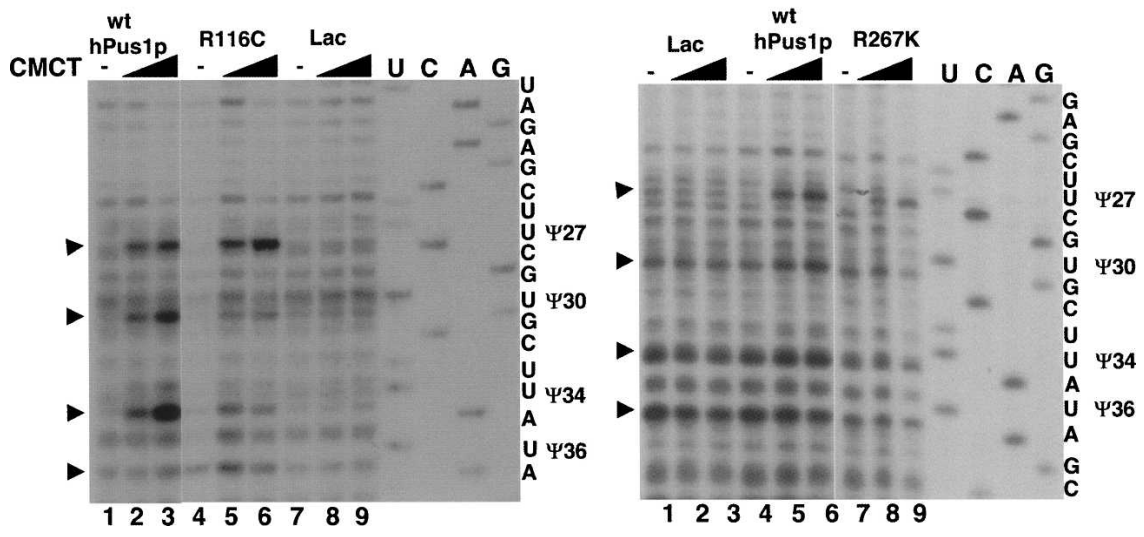

FIGURE 4. CMCT-primer extension assay of activity for wild-type and mutant hPuslp on $S$. cerevisiae pre-tRNA ${ }^{\mathrm{Il}}$. These assays were carried out on in vitro modified $S$. cerevisiae pretRNA $^{\text {Ile }}$ (UAU) incubated for three hours with either wild-type (wt), mutant (R116C $[A]$ or R267K $[B]$ ) hPus1p, or with Lac protein as described in Materials and Methods. The filled triangles denote an increase in CMCT concentration from 0.042 to $0.167 \mathrm{M} \mathrm{CMCT}$ in the chemical modification portion of the assay and a "-" denotes that no CMCT was included. Sequence lanes are deduced from chain terminating reactions using the same primer used in the CMCT assays, with the $S$. cerevisiae pre-tRNA $A_{U A U}^{\mathrm{Ile}}$ plasmid as the template. The uridines converted to $\Psi$ are noted to the right of each panel and the positions of the stops to reverse transcriptase just prior to those uridines are denoted on the left of each panel.

obtained for wild-type hPuslp with this substrate (see Tables 1,2). Interestingly, a $\Psi$ is indicated strongly at position 27 when the R116C mutant was used for modification, but the bands at the other positions (30 and 34) are not as intense as the bands in the wild-type hPuslp lanes (Fig. 4A, cf. lanes 2,3 and 5,6). This result is consistent with the value of $1.18 \mathrm{~mol} \Psi / \mathrm{mol}$ tRNA in the ${ }^{3} \mathrm{H}$-release data (Table 1) for this substrate with R116C.

In Figure 4B, a comparison of wild-type hPuslp and the $\mathrm{R} 267 \mathrm{~K}$ mutant shows the same result. There is a prominent band at position 27 (CMCT treated) in both the wild-type and $\mathrm{R} 267 \mathrm{~K}$ incubated lanes, and no band in the untreated lanes (Fig. 4B, cf. lanes 4-6 and 7-9). At position 30, with wild-type hPuslp, there is a band of increasing intensity in the CMCT-treated lanes (Fig. 4B, lanes 4-6), but with $\mathrm{R} 267 \mathrm{~K}$ there is little or no increase in the intensity of the band with CMCT treatment (Fig. 4B, lanes 7-9), indicating there is little, if any, $\Psi$ formed at this position. With wildtype hPuslp there is an increase in the intensity of the band at position 34 but there is no increase with the $\mathrm{R} 267 \mathrm{~K}$ mutant at this position. At position 36 the relatively high background coupled with the fact that the level of pseudouridylation at this position is low even with wild-type hPus1p precludes assaying the level of modification at this position. Nonetheless, it is obvious from the two panels that, although the mutants can modify the uridine at position 27, the other positions are left largely unmodified when compared with wild-type hPuslp.

The CMCT assay is only semi-quantitative and we wanted to measure the levels of $\Psi$ at all the positions modified by hPus1p in y pre-tRNA ${ }^{\text {Ile }}$. A 2D-TLC procedure was used to obtain a quantitative value for the amount of $\Psi$ formed at position 27, position 30, and positions 34 and 36 (combined) in $y$ pre-tRNA ${ }^{\text {Ile }}$ when hPus1p, a Lac control, and the R116A, R116K, R116S, Y173C, and R267K mutants were used in the modification reactions. The strategy for this method involves labeling the tRNA substrate used in the reactions with one of three $\alpha-{ }^{32}$ P-labeled NTPs. When the tRNA is digested with RNase $\mathrm{T}_{2}$ after incubation with the $\Psi$ synthases, the nucleotide $5^{\prime}$ to the labeled nucleotide will retain the labeled phosphate, effectively transferring the label and yielding a $3^{\prime}$-labeled nucleotide that can be chromatographed on thin-layer plates. By judicious selection of the labeled NTP used during the synthesis of the $y$ pretRNA ${ }^{\text {Ile }}$, we can determine the levels of $\Psi$ at the positions modified by hPuslp. Figure $5 \mathrm{~A}$ contains the images from the assay to determine $\Psi$ at position 27 . The five spots on the wild-type hPuslp panel represent the four nucleotides (NMPs, with a $3^{\prime}$ phosphate) and $\Psi \mathrm{p}$ on the tRNA that occur $5^{\prime}$ to a cytosine. A $\Psi$ spot is visible in every image except for Lac, indicating that all of the mutants and wild-type hPuslp formed $\Psi$ at position 27.

The spots in Figure 5B represent $\Psi$ s $5^{\prime}$ to an adenine; this includes positions 34 and 36, but will measure the level primarily at position 34 given the known activity of mPuslp (Behm-Ansmant et al. 2006) and what was shown for hPuslp in Figure 4. A $\Psi$ spot is clearly visible in only the chromatogram where wild-type hPuslp was incubated with the substrate. This indicates that none of the mutants tested in this assay retained significant activity at positions 34 or 36 and is consistent with what was shown earlier using the CMCT assay (Fig. 4). Figure $5 \mathrm{C}$ contains the images for determining the level of $\Psi$ at position 30. A $\Psi$ spot is visible on these overexposed TLCs when both wild-type hPuslp and the R267K mutant were used, though the $\Psi$ spot is much more apparent on the wild-type hPuslp chromatogram.

The intensity of the spots in the chromatograms shown in Figure 5 was measured using a phosphoimager. These data are presented as mol $\Psi / \mathrm{mol}$ tRNA and as the percent of wild-type hPus1p activity in Table 3. All of the mutants except Y173C showed near wild-type activity at position 27. None of the mutants showed substantial activity at positions 34 and 36, only R116A and R116K had any activity above zero. All of the mutants registered at least some minimal activity at position 30 with significant, but still low, activity exhibited by the R116K and R267K mutants. 
A

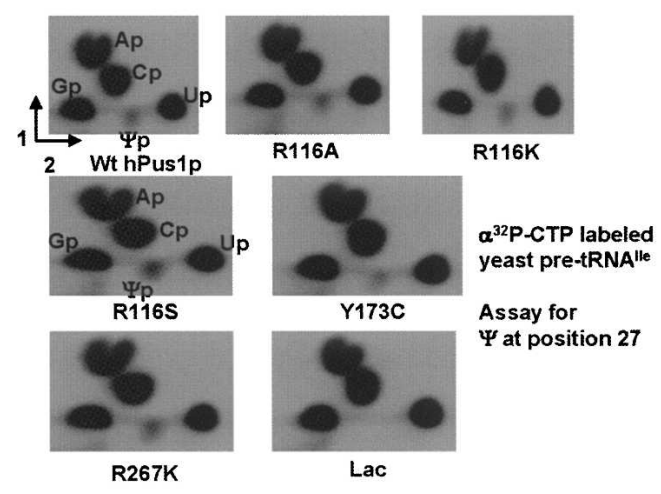

C

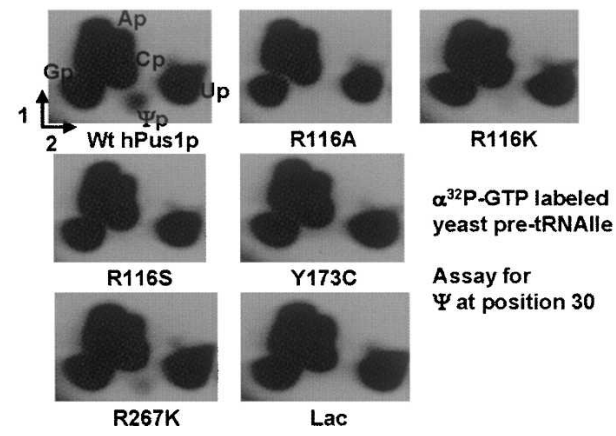

B

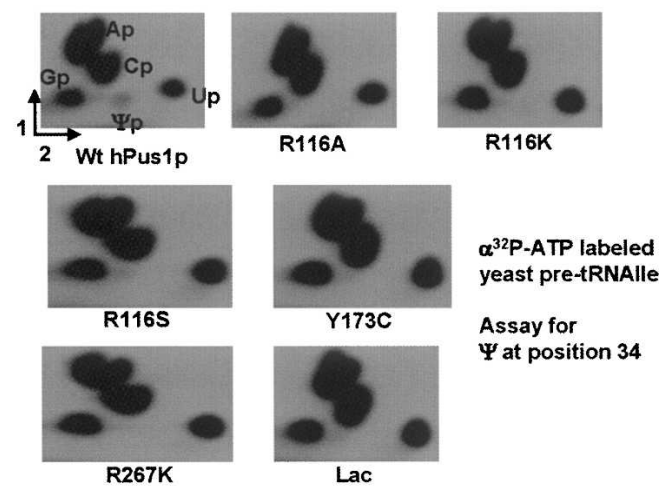

FIGURE 5. Assay of the level of modification at each position in S. cerevisiae pre-tRNA Ile $\mathrm{UAU}$ by wild-type and mutant hPuslps. Autoradiographs of portions of each of the two-dimensional TLCs are shown. Substrate tRNA, labeled with the nucleotide noted in the panel, $(A)$ position 27, ${ }^{32} \mathrm{P}$-CTP labeled, $(B)$ position $34,{ }^{32} \mathrm{P}$-ATP labeled, and $(C)$ position $30,{ }^{32} \mathrm{P}$-GTP labeled, was incubated with wild-type or mutant hPus1p for three hours, the RNA in the reaction isolated, gel-purified, digested with RNase $\mathrm{T}_{2}$, and chromatographed in two dimensions on cellulose TLC plates $(10 \mathrm{~cm} \times 10 \mathrm{~cm})$ as described in Materials and Methods. The position on pre-tRNA ${ }^{\text {Ile }}$ assayed is noted in each panel and the spots are identified in at least one chromatogram in each panel.

\section{DISCUSSION}

\section{Comparison of TruA and Pus1p}

TruA has been the focus of several structural studies and is the founding member of the family that includes Pus1p. These studies (Foster et al. 2000; Hur and Stroud 2007) provide a framework to discuss the present results, given the structure of Pus1p has not been solved from any species. There are some notable differences that have been observed for TruA and Puslp even though they are members of the same family. TruA is a homodimer in its tRNA-binding, active form (Foster et al. 2000), whereas $S$. cerevisiae Pus1p is not (Arluison et al. 1999a). Although TruA requires the entire tRNA as a substrate for modification (Hur et al. 2006), S. cerevisiae Puslp can modify a mini-substrate composed of an anticodon loop containing an intron (Motorin et al. 1998). TruA modifies only uridines found at positions 38,39 , and 40 in a number of Escherichia coli tRNAs, but Puslp modifies uridines at positions $1,26,27,28,30,34,36,65$, and 67 depending on the source of the enzyme ( $S$. cerevisiae or mouse), the substrates (intron-containing or not), or whether the activity was monitored in vivo or in vitro (Simos et al. 1996; Motorin et al. 1998; Chen and Patton 1999; BehmAnsmant et al. 2006). S. cerevisiae Pus1p has been shown to modify uridines at all the positions stated except position 30 (Motorin et al. 1998; Behm-Ansmant et al. 2006), and mouse Puslp has been shown to modify uridines at positions 1, 27, 28, 30, 34, and 36 (Chen and Patton 1999; Behm-Ansmant et al. 2006). Out of all the positions that Pus1p modifies, the modification of uridines at positions 27 and 28 is by far the most common in tRNAs, with $\Psi$ infrequently found at the other positions that the Puslp enzymes recognize (Sprinzl et al. 1998). Even with these differences between Puslp and TruA, the amino acids composing the active sites of these enzymes are highly conserved and the general mechanisms of substrate recognition and modification are probably comparable since all $\Psi$ synthases, in all families that have been identified thus far, have a core structure (for review, see Hur et al. 2006). 
TABLE 3. Quantitation from 2D TLCs of $S$. cerevisiae pre-tRNA $U$ Ue labeled with $\alpha^{32}$ P-NTP incubated with wild-type (wt) hPus $1 \mathrm{p}$ and various mutants

\begin{tabular}{|c|c|c|c|c|c|c|}
\hline \multirow[b]{2}{*}{ Enzyme } & \multicolumn{2}{|c|}{ CTP labeled $(\Psi \text { at } 27)^{\mathrm{a}}$} & \multicolumn{2}{|c|}{ ATP labeled ( $\Psi$ at 34,36$)$} & \multicolumn{2}{|c|}{ GTP labeled ( $\Psi$ at 30$)$} \\
\hline & $\frac{\operatorname{mol} \Psi}{\mathrm{mol} t \mathrm{RNA}}$ & Percent of wt $(\%)$ & $\frac{\mathrm{mol} \Psi}{\mathrm{mol} \text { tRNA }}$ & Percent of wt (\%) & $\frac{\operatorname{mol} \Psi}{\mathrm{mol} \text { tRNA }}$ & Percent of wt (\%) \\
\hline wt hPus1p & 0.76 & 100 & 0.92 & 100 & 0.45 & 100 \\
\hline R116A & 0.68 & 89 & 0.06 & 8 & 0.03 & 7 \\
\hline R116K & 0.75 & 99 & 0.08 & 9 & 0.05 & 11 \\
\hline R116S & 0.79 & 104 & 0.00 & 0 & 0.02 & 4 \\
\hline Y173C & 0.38 & 50 & 0.00 & 0 & 0.01 & 2 \\
\hline R267K & 0.71 & 93 & 0.00 & 0 & 0.10 & 22 \\
\hline
\end{tabular}

Activity is reported in $\mathrm{mol} \Psi / \mathrm{mol}$ tRNA and as the percentage of wt hPus $1 \mathrm{p}$ at that position.

aThe positions of $\Psi$ that are possible Pus $1 p$ modification sites given the nucleotide label are given next to the labeled nucleotide in the column heading. The counts were measured on the TLC plates using a Phosphorlmager. The activity in mol $\Psi / \mathrm{mol}$ tRNA has had the value measured for incubation of the substrate for the same length of time with bacterially expressed Lac subtracted. A value of " 0.00 " indicates that there was no activity above background observed.

\section{Arginines and tyrosine in the active site}

Foster et al. (2000) showed that the arginines at positions 58 and 205 in TruA (equivalent to 116 and 267 in hPuslp) interact with the active site aspartate (D60 in TruA, D118 in hPus1p) through bridging water molecules. Mutation of R58 to alanine in TruA results in an inactive enzyme that still binds tRNA with a similar affinity as wild-type TruA (Hur and Stroud 2007) and led these investigators to conclude that R58 facilitates base-flipping rather than taking part in the recognition or catalysis steps (Hur and Stroud 2007). The equivalent position (R62) in RluA also appears to have a key role in the flipping of the target base (Hoang et al. 2006) and, when R62 was replaced with either lysine or methionine, all activity was lost in RluA (Hoang et al. 2006). Given that patients with MLASA have a mutation at the equivalent position in hPuslp (R116W) that also renders the enzyme inactive (Bykhovskaya et al. 2004; Patton et al. 2005), one would assume that there would be little tolerance for substitution at this position. Our data show that in fact several of the hPuslp mutants at position 116 exhibit significant activity, especially when the modification at positions 27 or 28 is considered. The levels of modification at additional sites such as 30 and 34 are greatly diminished, but that "core" activity at $27 / 28$ is retained, albeit with an increased $K_{m}$ and slightly reduced affinity for the substrate. The same is true for mutations at other positions (Y173 and R267) in hPus1p; some mutations result in no activity, such as Y173S, but others show some activity toward modification at position 27 in the $S$. cerevisiae pre-tRNA ${ }^{\text {Ile }}$. When the corresponding tyrosine in E. coli TruB (Y67) was mutated to phenylalanine, leucine, or alanine, there was no detectable activity on a natural tRNA substrate (Phannachet et al. 2005), suggesting that there was a need for both a hydrophobic ring and an $\mathrm{OH}$ group combined in one side chain (Phannachet et al. 2005). These data would suggest that the functions of these highly conserved amino acids are less constrained in hPuslp than for instance R62 in RluA, R58 in TruA, and Y67 in TruB. The substitution of R116 with glutamic acid, histidine, or tryptophan is not tolerated in hPuslp because of either steric or charge constraints, but there are not strict requirements for the presence of arginine at this position in hPuslp, at least when the modification of position $27 / 28$ is considered.

If R116 and R267 were essential to the catalytic function of hPuslp we would expect to see an approximately equal decrease in activity at all positions on the substrate. However, all of the mutants retained activity almost exclusively at position 27 or 28 . This confirms that the role of the two arginines is more likely one of substrate manipulation. As mentioned previously, by comparison with TruA, the putative role of R116 in hPuslp is to flip the uridine out of the RNA and into the active site of the enzyme and at the same time stabilize the RNA structure by substituting for the flipped out uridine (Hur and Stroud 2007). We would not expect most of the smaller side chain amino acid mutations, especially glycine, to be able to help fulfill either of these roles. It is possible that the uridines at positions 27 and 28 are especially prone to flipping out of the RNA. Perhaps, since they are at the end of the stem, they are not as stably held as others in the middle of a stem might be, for instance a uridine at position 30 .

One could reasonably expect to retain some enzymatic activity when replacing an arginine with lysine, because the two molecules are very similar in size, shape, and charge. This explains why both $\mathrm{R} 116 \mathrm{~K}$ and $\mathrm{R} 267 \mathrm{~K}$ were among the mutants with the highest levels of retained activity. They might participate weakly in base flipping and also replace the displaced uridine. The polar side chain of cysteine is much smaller than lysine, has no charge at neutral $\mathrm{pH}$, and has a different functional group ( $\mathrm{SH})$, so it is not immediately apparent why the R116C mutant was able to retain activity levels equivalent to R116K. Mutants of R116 with other smaller amino acid side chains such as alanine, glycine, asparagine, and serine all retained some activity, 
presumably because, while they might not actively participate in flipping out or replacement of the uridine, neither will they sterically block the active site, as R116W might.

In terms of Y173, it has been suggested that, at least in TruB, the corresponding tyrosine (Y67 in E. coli TruB) is involved in maintaining the catalytic aspartate in the charged state through the maintenance of a salt bridge (Hoang and Ferré-D'Amaré 2001; Chaudhuri et al. 2004). Our results with mutations at this position suggest that a tyrosine at this position is indeed critical, but there was detectable activity with all the mutants except Y173S. The two mutants with the highest activity are Y173C and Y173T, both with side chains that could participate in the interaction with the aspartate in the active site, although cysteine has an ionizable side ( $\mathrm{pK} 8.3$ ) chain like tyrosine (pK 10), whereas threonine does not. Why there is detectable activity with the Y173G and Y173F mutants, whose side chains would not be expected to participate in this salt bridge, is unknown. In addition, no activity was seen with Y173S, even though there is an $-\mathrm{OH}$ available for interaction with the aspartate. The particular characteristics of the tyrosine side chain, hydrophobicity of the phenyl ring, and an $\mathrm{OH}$ group with a $\mathrm{pK}$ of 10 appear to be required for the maintenance of the structure of the active site and full activity.

\section{Modification of uridine at position 30}

It has been shown that, when mPuslp is expressed in a $\Delta$ pus1 yeast strain, position 30 in y pre-tRNA $A_{U A U}^{\text {Ile }}$ is modified to $\Psi$. The activity was also observed in vitro with the same substrate and mPus1p, whereas S. cerevisiae Pus1p does not exhibit this activity in vivo or in vitro (Behm-Ansmant et al. 2006). In this study we observed that wild-type hPuslp can modify position 30 in y pre-tRNA ${ }^{\mathrm{I}-}$ le UAU (Fig. 4) and $\mathrm{m}$ tRNA $\mathrm{UAU}_{\text {Ile }}$ (Table 1) in vitro, but probably not at position 30 in h pre-tRNA $A_{A A U}^{\mathrm{Ile}}$. However, modification of uridine at this position is severely hampered by mutation at any of the three active site residues.

\section{Milder phenotypes anticipated with some mutations and possible recovery of activity}

Since the positions that were chosen for mutation are highly conserved in $\Psi$ synthases, it was somewhat surprising to find that any substitution is tolerated. In fact some substitutions at these residues resulted in proteins with nearly a wild-type level of activity at sites 27 and 28 on tRNAs. The R116W mutation seen in MLASA patients may lead to the most severe phenotype and milder phenotypes might occur if a different amino acid was substituted at this position. The codon used to code for arginine at position 116 in humans is CGG and point mutations in the first two positions of the triplet can give rise to five amino acid substitutions, glycine, glutamine, leucine, proline, and tryptophan. The R116G and R116Q mutants had activities on the level of the R116A mutant and so it is possible that milder phenotypes might result from such mutations.

A recent report showed that enzymatic activity could be recovered in an inactive mutant kinase by the treatment of cells in culture containing the mutant enzyme with small molecules that mimicked the chemistry of the lost amino acid side chain (Qiao et al. 2006). The kinase (proteintyrosine kinase C-terminal Src kinase) has an active site that pairs an aspartate with a nearby arginine (four residues away) and looks remarkably like the active site of Puslp. Earlier work had shown that chemical rescue of a mutant enzyme, that replaces the arginine with alanine, was possible and that the class of small molecules that might restore activity could be predicted given knowledge of the composition of the active site (Williams et al. 2000; Zhao et al. 2004a). By understanding the topography of the active site of hPuslp and determining the flexibility of the requirements for particular amino acids that compose it, the pathway to identifying a class of compounds that could be considered for recovery of mutant hPuslp activity might be less uncertain. With the extensive small molecule libraries that are available and the high throughput methods for the screening of these compounds that have been developed, it is now reasonable to assume that a substance might be identified that will restore partial function to the R116W mutant hPus1p or perhaps restore complete activity to other mutants at this or other positions.

\section{MATERIALS AND METHODS}

\section{Creation of wild-type and mutant hPus1p}

A human PUS1 cDNA fragment was generated from the I.M.A.G.E. consortium (Lennon et al. 1996) clone \#2822709 (obtained from the American Type Culture Collection) using PCR (Mullis et al. 1986) with primers HuPus1pET16bFor (5' AACATATGGCCGGGAACGCGGAGCC) and HuPus1pET16bRev (5'-GGATCCTCGAGTCCCATCGCCTCAGTCAGTG) as described (Chen and Patton 1999). The PCR fragment was inserted into the pGEMT (Promega) vector and sequenced (Sanger et al. 1977). The fragment was cut from pGEMT with Nde1 and Xho1, gel-purified, and ligated into pET16b plasmid DNA (Novagen) cut with Ndel and Xhol. The purified hPuslppET16b DNA was sequenced, used to transform pLysS (DE3; Promega) cells, and the recombinant hPuslp expressed and isolated as described for mPus1p (Chen and Patton 1999). As with mPus1p, there are an additional 21 amino acids at the amino terminus of hPuslp encoded by the vector (MGHHHHHHHH HHSSGHIEGRH), which are used for purification.

Site-directed mutagenesis was performed with a Quikchange II kit (Stratagene), utilizing the primers listed below, and the hPuslppET16b plasmid, to create the hPuslp mutants. The insert was sequenced to confirm the mutation and to make sure no additional mutations occurred. The recombinant mutant proteins were isolated as described (Chen and Patton 1999) except that $\beta$-mercaptoethanol was used in the dialysis buffer instead of dithiothreitol; it lowers the amount of precipitation of the protein during dialysis. 
Primers 5'-GCGCTGCGCCGCCACAGACAAGGG and 5'-CCC TTGTCTGGCGGCGCAGCGC were used to change R116 to alanine;

Primers 5'-GCGCTGCGCCAACACAGACAAGGG and 5' -CCCT TGTCTGTGTTGGCGCAGCGC to change R116 to asparagine;

Primers 5'-GCGCTGCGCCTGCACAGACAAGGG and 5'-CCC TTGTCTGTGCAGGCGCAGCGC to change R116 to cysteine;

Primers 5'-GCGCTGCGCCGGCACAGACAAGGG and 5' -CCCT TGTCTGTGCCGGCGCAGCGC to change R116 to glycine;

Primers 5'-GCGCTGCGCCGAAACAGACAAGGG and 5'-CCCT TGTCTGTTTCGGCGCAGCGC to change R116 to glutamic acid;

Primers 5'-GCGCTGCGCCCAGACAGACAAGGG and 5' -CCCT TGTCTGTCTGGGCGCAGCGC to change R116 to glutamine;

Primers 5'-GCGCTGCGCCCATACAGACAAGGG and 5'-CCCT TGTCTGTATGGGCGCAGCGC to change R116 to histidine;

Primers 5'-GCGCTGCGCCAAAACAGACAAGGG and 5' -CCCT TGTCTGTTTTGGCGCAGCGC were used to change R116 to lysine;

Primers 5'-GCGCTGCGCCAGCACAGACAAGGG and 5' -CCCT TGTCTGTGCTGGCGCAGCGC to change R116 to serine;

Primers 5'-CAGCGCTGCGCCTGGACAGACAAGG and 5'-CCT TGTCTGTCCAGGCGCAGCGCTG were used to change the arginine at 116 to tryptophan;

Primers 5'-GTGATGCCAGGACCTGCTGCTACCTGCTGCC and 5'-GGCAGCAGGTAGCAGCAGGTCCTGGCATCAC to change Y173 to cysteine;

Primers 5'-GTGATGCCAGGACCGGCTGCTACCTGCTGCC and 5'-GGCAGCAGGTAGCAGCCGGTCCTGGCATCAC to change Y173 to glycine;

Primers 5'-GTGATGCCAGGACCTTCTGCTACCTGCTGCC and 5'-GGCAGCAGGTAGCAGAAGGTCCTGGCATCAC to change Y173 to phenylalanine;

Primers 5'-GTGATGCCAGGAACCAGCTGCTACCTGCTGCCC and $5^{\prime}$-GGGCAGCAGGTAGCAGCTGGTCCTGGCATCAC to change Y173 to serine;

Primers 5'-GTGATGCCAGGACCACCTGCTACCTGCTGCCC and 5'-GGGCAGCAGGTAGCAGGTGGTCCTGGCCATCAC to change Y173 to threonine;

Primers 5'-GATGCATCAGATCGGCAAGATGGTCGGCCTGG and 5'-CCAGGCCGACCATCTTGCCGATCTGATGCATC were used to convert R267 to glycine; and

Primers 5' -GATGCATCAGATCAAAAAGATGGTCGGCCTGG and 5'-CCAGGCCGACCATCTTTTTGATCTGATGCATC were used to convert R267 to lysine.

\section{tRNA substrate cloning}

The tRNA genes for cytoplasmic m pre-tRNA Ile $h$ tRNA Ale ${ }_{\text {AAU }}^{\text {Ile }}$ were amplified from mouse or human DNA using PCR (Mullis et al. 1986) and the following primers for $m$

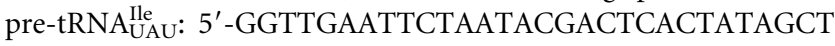
CCAGTGGCGCAATCGG and 5'-GGTTGGATCCACCATGGT GCTCCAGGTGAGGCTCGAAC; and the following primers for $h$ tRNA $^{\text {Ile }}$ (AAU): 5'-GGTTGAATTCTAATACGACTCACTATAGG CCGGTTAGCTTCAGTTGG and 5'-GGTTGGATCCTGGTGGC CAGTACGGGGATCG. The gene for mouse cytoplasmic tRNA ${ }^{\text {Met }}$ was amplified from mouse genomic DNA using the following primers: 5'-GGATGAATTCTAATACGACTCACTATAGCCTCG TTAGCGCAGTAGG and 5'-CCATGGATCCTGGTGCCCCGTG
TGAGGATCG. The resulting fragments were digested with BamH1 and EcoR1, gel-purified, and ligated into gel-purified pUC19 plasmid DNA digested with BamH1 and EcoR1. The resulting constructs have a T7 RNA polymerase promoter at the $5^{\prime}$ end of the tRNA gene and a BstN1 site at the $3^{\prime}$ end of the gene so that plasmids digested with BstN1 will yield tRNAs with CCA at the $3^{\prime}$ terminus after transcription.

\section{RNA synthesis and tritium release assay}

The plasmid DNAs containing the human serine tRNA (h tRNA $^{\text {Ser }}$ ) gene (gift of H. Gross, Würzburg, Germany) and the S. cerevisiae pre-tRNA ${ }^{\text {Ile }}$ (y pre-tRNA ${ }^{\text {Ile }}$ ) (gift of H. Grosjean, Orsay, France, and F. Fasiolo, Strasbourg, France) as well as DNAs for $m$ pre-tRNA $\mathrm{Ile}, \mathrm{h}$ tRNA $\mathrm{AAU}_{\mathrm{Al}}^{\mathrm{Ile}}$, and $\mathrm{m} \mathrm{tRNA}^{\text {Met }}$ were digested with BstN1 and transcribed $(25 \mu \mathrm{L}$ reactions) with T7 RNA polymerase (Promega Corp.) as described, in the presence of 25 $\mu \mathrm{Ci}\left[5-{ }^{3} \mathrm{H}\right]-\mathrm{UTP}(20 \mathrm{Ci} / \mathrm{mmol}$; Moravek Biochemicals and Radiochemicals) with $100 \mu \mathrm{M}$ cold UTP and $1.0 \mathrm{mM}$, ATP, GTP, and CTP (Chen and Patton 1999).

Before incubation with wild-type or mutant hPuslp or a Lac control protein expressed in bacteria and isolated in the same way as the hPus1p proteins (Chen and Patton 1999), the RNA substrates were heated to $78^{\circ} \mathrm{C}$ for $2 \mathrm{~min}$ and allowed to cool slowly to $37^{\circ} \mathrm{C}$ (30 to $40 \mathrm{~min}$ ). Substrate reactions with the $\Psi$ synthases were carried out in $100 \mathrm{mM}$ ammonium chloride, $10 \mathrm{mM}$ DTT, $50 \mathrm{mM}$ Tris ( $\mathrm{pH} 7.5$ ), and $2 \mathrm{mM} \mathrm{MgCl}_{2}$ (typically, $100 \mu \mathrm{L}$ reactions for each assay). The concentration of $\Psi$ synthase used was $\sim 40-50$ $\mathrm{nM}$. The lengths of incubation are listed in the text or in the tables containing the data. The tritium release assay method is described in detail elsewhere (Cortese et al. 1974; Patton 1991).

Short-term kinetic analysis of wild-type and R116K hPuslp was carried out as described (Arluison et al. 1999b) at ${ }^{3} \mathrm{H}-\mathrm{UTP}-$ labeled substrate (y pre-tRNA ${ }^{\text {Ile }}$ ) concentrations of between 10 and $50 \mathrm{nM}$ and at an enzyme concentration of $\sim 700 \mathrm{nM}$. Aliquots $(10 \mu \mathrm{L}$ from a $25 \mu \mathrm{L}$ total) were removed after 1- and 2-min incubation at $37^{\circ} \mathrm{C}$ and immediately mixed with charcoal to stop the reaction and allow for determination of the ${ }^{3} \mathrm{H}$ released. The specific activity of the ${ }^{3} \mathrm{H}$-labeled y pre-tRNA ${ }^{\text {Ile }}$ was changed for these experiments, in that the concentration of the cold UTP was $250 \mu \mathrm{M}$, and everything else was as listed above for the other ${ }^{3} \mathrm{H}$-labeled substrates. The results reported are from two independent assays. The binding studies were carried out as described (Arluison et al. 1998) using protein concentrations from 20 to $800 \mathrm{nM}$ in $100 \mu \mathrm{L}$ reactions and the ${ }^{3} \mathrm{H}$-labeled y pre-tRNA ${ }^{\mathrm{Ile}}$ substrate at $500 \mathrm{pM}$.

\section{CMCT Pseudouridine mapping assay}

The method for the mapping of $\Psi$ residues has been described previously (Bakin and Ofengand 1998; Baumstark and Ahlquist 2001). Three-hour incubations were performed using the ${ }^{3} \mathrm{H}$-labeled y pre-tRNA ${ }^{\text {Ile }}$ substrate with wild-type hPus1p, the R116C and R267K mutants, and a Lac control as described above. A small aliquot was removed to measure the overall progress of the reaction, using the ${ }^{3} \mathrm{H}$-release assay. The RNA in each reaction was isolated and was treated with either $0,0.042 \mathrm{M}$, or $0.167 \mathrm{M}$ CMCT (1cyclohexyl-3[2-morpholinoethyl] carbodiimide metho-p-toluenesulfonate, Aldrich). After treatment with sodium carbonate $(4 \mathrm{~h}$, $37^{\circ} \mathrm{C}$ ) to remove all the adducts except those attached to $\Psi$ residues, the RNA samples were used as templates for reverse transcription 
using AMV reverse transcriptase (Promega) and a ${ }^{32} \mathrm{P}$-end-labeled primer ( 5 '-CACAGAAACTTCGGAAACCG). The fmole Sequencing kit (Promega) was used to generate the sequencing lanes for the gels, using y pre-tRNA ${ }^{\text {Ile }}$ plasmid DNA as the template.

\section{Thin layer chromatography assay of $\Psi$ formation}

To determine the levels of $\Psi$ at positions $27,30,34$, and 36 in y pretRNA $^{\text {Ile }}$ the substrate was synthesized as above but $\alpha-{ }^{32} \mathrm{P}-\mathrm{NTPs}(50$ $\mu \mathrm{Ci}, 3000 \mathrm{Ci} / \mathrm{mmol}, 25 \mu \mathrm{L}$ reactions; MP Biomedicals) were used in place of the ${ }^{3} \mathrm{H}$-UTP. To determine levels of $\Psi$ at these positions, the substrate was labeled with $\alpha-{ }^{32} \mathrm{P}-\mathrm{CTP}$ (for position 27), $\alpha-{ }^{32} \mathrm{P}-$ GTP (for position 30), or $\alpha-{ }^{32} \mathrm{P}-\mathrm{ATP}$ (for positions 34 and 36). The concentration of the nucleotides was $1.0 \mathrm{mM}$, except for the labeled nucleotide, which was $100 \mu \mathrm{M}$. The modification reactions were set up as above and the RNA isolated after a 3-h incubation. This isolated RNA was electrophoresed on a denaturing $10 \%$ polyacrylamide gel (19:1; 8.3 M urea) and the full-length RNA was eluted from the gel (Sambrook and Rusell 2001). The gel-purified RNA was digested with $\mathrm{RNase}_{2}$, prepared as described (Hiramaru et al. 1966; Lichtler et al. 1992), and chromatographed on thin layer plates (cellulose, $10 \mathrm{~cm} \times 10 \mathrm{~cm}$ ) using isobutyric acid:ammonium hydroxide:water (66:1:33; v:v:v) in the first dimension and isopropanol:concentrated HCl:water (70:15:15;v:v:v) in the second (Grosjean et al. 2007). The dried TLC plates were exposed to Xray film to generate an image and the levels of ${ }^{32} \mathrm{P}$ in the spots quantified with a Personal FX PhosphoImager (Bio-Rad).

\section{ACKNOWLEDGMENTS}

The plasmid for the synthesis of human tRNA ${ }_{U G A}^{\text {Ser }}$ was a gift of $H$. Gross and the plasmid for the synthesis of $S$. cerevisiae pre-tRNA ${ }^{\text {Ile }}$ was a gift of H. Grosjean and F. Fasiolo. We thank the National Institutes of Health (DK074368-01) for funding and the South Carolina Honors College at the University of South Carolina for an Undergraduate Research Fellowship to B.S.S.

Received January 2, 2008; accepted May 12, 2008.

\section{REFERENCES}

Arluison, V., Hountondji, C., Robert, B., and Grosjean, H. 1998. Transfer RNA-pseudouridine synthetase Pus1 of Saccharomyces cerevisiae contains one atom of zinc essential for its native conformation and tRNA recognition. Biochemistry 37: 7268-7276.

Arluison, V., Batelier, G., Ries-Kautt, M., and Grosjean, H. 1999a. RNA:pseudouridine synthetase Pus1 from Saccharomyces cerevisiae: Oligomerization property and stoichiometry of the complex with yeast tRNA ${ }^{\text {Phe }}$. Biochimie 81: 751-756.

Arluison, V., Buckle, M., and Grosjean, H. 1999b. Pseudouridine synthetase Pus1 of Saccharomyces cerevisiae: Kinetic characterisation, tRNA structural requirement and real-time analysis of its complex with tRNA. J. Mol. Biol. 289: 491-502.

Auffinger, P. and Westhof, E. 1998. Appendix 5: Location and distribution of modified nucleotides in tRNA. In Modification and editing of RNA (eds. H. Grosjean and R. Benne), pp. 569-576. ASM Press, Washinton, D.C.

Bakin, A.V. and Ofengand, J. 1998. Mapping of pseudouridine residues in RNA to nucleotide resolution. Methods Mol. Biol. 77: 297-309.

Baumstark, T. and Ahlquist, P. 2001. The brome mosaic virus RNA3 intergenic replication enhancer folds to mimic a tRNA TpsiC-stem loop and is modified in vivo. RNA 7: 1652-1670.
Behm-Ansmant, I., Massenet, S., Immel, F., Patton, J.R., Motorin, Y., and Branlant, C. 2006. A previously unidentified activity of yeast and mouse RNA:pseudouridine synthases 1 (Pus1p) on tRNAs. RNA 12: 1583-1593.

Bjork, G.R., Ericson, J.U., Gustafsson, C.E., Hagervall, T.G., Jonsson, Y.H., and Wikstrom, P.M. 1987. Transfer RNA modification. Annu. Rev. Biochem. 56: 263-287.

Bykhovskaya, Y., Casas, K., Mengesha, E., Inbal, A., and FischelGhodsian, N. 2004. Missense mutation in pseudouridine synthase 1 (PUS1) causes mitochondrial myopathy and sideroblastic anemia (MLASA). Am. J. Hum. Genet. 74: 1303-1308.

Chaudhuri, B.N., Chan, S., Perry, L.J., and Yeates, T.O. 2004. Crystal structure of the apo forms of $\Psi 55$ tRNA pseudouridine synthase from Mycobacterium tuberculosis: A hinge at the base of the catalytic cleft. J. Biol. Chem. 279: 24585-24591.

Chen, J. and Patton, J.R. 1999. Cloning and characterization of a mammalian pseudouridine synthase. RNA 5: 409-419.

Chen, J. and Patton, J.R. 2000. Mouse pseudouridine synthase 1: Gene structure and alternative splicing of pre-mRNA. Biochem. J. 352: 465-473.

Conrad, J., Niu, L., Rudd, K., Lane, B.G., and Ofengand, J. 1999. 16 S ribosomal RNA pseudouridine synthase RsuA of Escherichia coli: Deletion, mutation of the conserved Asp102 residue, and sequence comparison among all other pseudouridine synthases. RNA 5: 751-763.

Cortese, R., Kammen, H.O., Spengler, S.J., and Ames, B.N. 1974. Biosynthesis of pseudouridine in transfer ribonucleic acid. J. Biol. Chem. 249: 1103-1108.

Davis, D.R. 1995. Stabilization of RNA stacking by pseudouridine. Nucleic Acids Res. 23: 5020-5026.

Davis, D.R. and Poulter, C.D. 1991. 1H-15N NMR studies of Escherichia coli tRNA(Phe) from hisT mutants: A structural role for pseudouridine. Biochemistry 30: 4223-4231.

Davis, D.R., Veltri, C.A., and Nielsen, L. 1998. An RNA model system for investigation of pseudouridine stabilization of the codonanticodon interaction in tRNALys, tRNAHis and tRNATyr. $J$. Biomol. Struct. Dyn. 15: 1121-1132.

Del Campo, M., Kaya, Y., and Ofengand, J. 2001. Identification and site of action of the remaining four putative pseudouridine synthases in Escherichia coli. RNA 7: 1603-1615.

Donmez, G., Hartmuth, K., and Luhrmann, R. 2004. Modified nucleotides at the $5^{\prime}$ end of human U2 snRNA are required for spliceosomal E-complex formation. RNA 10: 1925-1933.

Foster, P.G., Huang, L., Santi, D.V., and Stroud, R.M. 2000. The structural basis for tRNA recognition and pseudouridine formation by pseudouridine synthase I. Nat. Struct. Biol. 7: 23-27.

Grosjean, H., Droogmans, L., Roovers, M., and Keith, G. 2007. Detection of enzymatic activity of transfer RNA modification enzymes using radiolabeled tRNA substrates. Methods Enzymol. 425: $55-101$.

Hamma, T. and Ferré-D’Amaré, A.R. 2006. Pseudouridine synthases. Chem. Biol. 13: 1125-1135.

Hiramaru, M., Uchida, T., and Egami, F. 1966. Ribonuclease preparation for the base analysis of polyribonucleotides. Anal. Biochem. 17: 135-142.

Hoang, C. and Ferré-D’Amaré, A.R. 2001. Cocrystal structure of a tRNA $\Psi 55$ pseudouridine synthase: Nucleotide flipping by an RNA-modifying enzyme. Cell 107: 929-939.

Hoang, C., Chen, J., Vizthum, C.A., Kandel, J.M., Hamilton, C.S., Mueller, E.G., and Ferré-D’Amaré, A.R. 2006. Crystal structure of pseudouridine synthase RluA: Indirect sequence readout through protein-induced RNA structure. Mol. Cell 24: 535-545.

Huang, L., Pookanjanatavip, M., Gu, X., and Santi, D.V. 1998. A conserved aspartate of tRNA pseudouridine synthase is essential for activity and a probable nucleophilic catalyst. Biochemistry 37: 344-351.

Hur, S. and Stroud, R.M. 2007. How U38, 39, and 40 of many tRNAs become the targets for pseudouridylation by TruA. Mol. Cell 26: 189-203. 
Hur, S., Stroud, R.M., and Finer-Moore, J. 2006. Substrate recognition by RNA 5-methyluridine methyltransferases and pseudouridine synthases: A structural perspective. J. Biol. Chem. 281: 38969-38973.

Kaya, Y. and Ofengand, J. 2003. A novel unanticipated type of pseudouridine synthase with homologes in bacteria, archaea, and eukarya. RNA 9: 711-721.

Koonin, E.V. 1996. Pseudouridine synthases: Four families of enzymes containing a putative uridine-binding motif also conserved in dUTPases and dCTP deaminases. Nucleic Acids Res. 24: 24112415.

Lennon, G., Auffray, C., Polymeropoulos, M., and Soares, M.B. 1996. The I.M.A.G.E. Consortium: An integrated molecular analysis of genomes and their expression. Genomics 33: 151-152.

Lichtler, A., Barrett, N.L., and Carmichael, G.G. 1992. Simple, inexpensive preparation of T1/T2 ribonuclease suitable for use in RNase protection experiments. Biotechniques 12: 231-232.

Massenet, S.A., Mougin, A., and Branlant, C. 1998. Posttranscriptional modifications of the U small nuclear RNAs. In Modification and editing of RNA (eds. H. Grosjean and R. Benne), pp. 201-227. ASM Press, Washington, D.C.

Massenet, S., Motorin, Y., Lafontaine, D.L., Hurt, E.C., Grosjean, H., and Branlant, C. 1999. Pseudouridine mapping in the Saccharomyces cerevisiae spliceosomal U small nuclear RNAs (snRNAs) reveals that pseudouridine synthase puslp exhibits a dual substrate specificity for U2 snRNA and tRNA. Mol. Cell. Biol. 19: 2142 2154.

Motorin, Y., Keith, G., Simon, C., Foiret, D., Simos, G., Hurt, E., and Grosjean, H. 1998. The yeast tRNA:pseudouridine synthase Puslp displays a multisite substrate specificity. RNA 4: 856-869.

Mullis, K., Faloona, F., Scharf, S., Saiki, R., Horn, G., and Erlich, H. 1986. Specific enzymatic amplification of DNA in vitro: The polymerase chain reaction. Cold Spring Harb. Symp. Quant. Biol. 51: $263-273$.

Ofengand, J. 2002. Ribosomal RNA pseudouridines and pseudouridne synthases. FEBS Lett. 514: 17-25.

Patton, J.R. 1991. Pseudouridine modification of U5 RNA in ribonucleoprotein particles assembled in vitro. Mol. Cell. Biol. 11: 5998-6006.

Patton, J.R. and Padgett, R.W. 2003. Caenorhabditis elegans pseudouridine synthase 1 activity in vivo: tRNA is a substrate but not U2 small nuclear RNA. Biochem. J. 372: 595-602.

Patton, J.R., Bykhovskaya, Y., Mengesha, E., Bertolotto, C., and Fischel-Ghodsian, N. 2005. Mitochondrial myopathy and sideroblastic anemia (MLASA): Missense mutation in the pseudouridine synthase 1 (PUS1) gene is associated with the loss of tRNA pseudouridylation. J. Biol. Chem. 280: 19823-19828.

Phannachet, K., Elias, Y., and Huang, R.H. 2005. Dissecting the roles of a strictly conserved tyrosine in substrate recognition and catalysis by pseudouridine 55 synthase. Biochemistry 44: 1548815494.

Qiao, Y., Molina, H., Pandey, A., Zhang, J., and Cole, P.A. 2006. Chemical rescue of a mutant enzyme in living cells. Science 311: 1293-1297.

Ramamurthy, V., Swann, S.L., Paulson, J.L., Spedaliere, C.J., and Mueller, E.G. 1999. Critical aspartic acid residues in pseudouridine synthases. J. Biol. Chem. 274: 22225-22230.

Raychaudhuri, S., Niu, L., Conrad, J., Lane, B.G., and Ofengand, J. 1999. Functional effect of deletion and mutation of the Escherichia coli ribosomal RNA and tRNA pseudouridine synthase RluA. J. Biol. Chem. 274: 18880-18886.

Sambrook, J. and Rusell, D.W. 2001. Molecular cloning: A laboratory manual. Cold Spring Harbor Laboratory Press, Cold Spring Harbor, NY.

Sanger, F., Nicklen, S., and Coulson, A.R. 1977. DNA sequencing with chain-terminating inhibitors. Proc. Natl. Acad. Sci. 74: 5463-5467.

Simos, G., Tekotte, H., Grosjean, H., Segref, A., Sharma, K., Tollervey, D., and Hurt, E.C. 1996. Nuclear pore proteins are involved in the biogenesis of functional tRNA. EMBO J. 15: 22702284.

Sprinzl, M., Horn, C., Brown, M., Ioudovitch, A., and Steinberg, S. 1998. Compilation of tRNA sequences and sequences of tRNA genes. Nucleic Acids Res. 26: 148-153.

Williams, D.M., Wang, D., and Cole, P.A. 2000. Chemical rescue of a mutant protein-tyrosine kinase. J. Biol. Chem. 275: 38127-38130.

Yu, Y.T., Shu, M.D., and Steitz, J.A. 1998. Modifications of U2 snRNA are required for snRNP assembly and pre-mRNA splicing. EMBO J. 17: 5783-5795.

Zebarjadian, Y., King, T., Fournier, M.J., Clarke, L., and Carbon, J. 1999. Point mutations in yeast CBF5 can abolish in vivo pseudouridylation of rRNA. Mol. Cell. Biol. 19: 7461-7472.

Zhao, X. and Yu, Y.-T. 2004. Pseudouridines in and near the branch site recognition region of $\mathrm{U} 2$ snRNA are required for snRNP biogenesis and pre-mRNA splicing in Xenopus oocytes. RNA 10: 681-690.

Zhao, L., Liao, H., and Tsai, M.D. 2004a. The catalytic role of aspartate in a short strong hydrogen bond of the Asp274-His32 catalytic dyad in phosphatidylinositol-specific phospholipase C can be substituted by a chloride ion. J. Biol. Chem. 279: 31995-32000.

Zhao, X., Patton, J.R., Davis, S.L., Florence, B., Ames, S.J., and Spangaard, R.A. 2004b. Regulation of nuclear receptor activity by a pseudouridine synthase through posttranscriptional modification of steriod receptor RNA activator. Mol. Cell 15: 549-558.

Zhao, X., Patton, J.R., Ghosh, S.K., Fischel-Ghodsian, N., Shen, L., and Spanjaard, R.A. 2007. Pus3p and Puslp-dependent pseudouridylation of steroid receptor RNA activator controls a functional switch that regulates nuclear receptor signaling. Mol. Endocrinol. 21: $686-699$. 

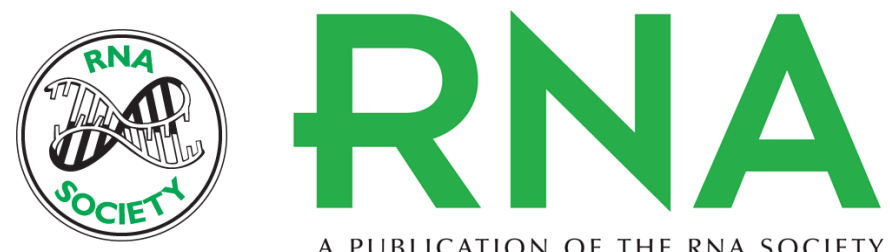

A PUBLICATION OF THE RNA SOCIETY

\section{Partial activity is seen with many substitutions of highly conserved active site residues in human Pseudouridine synthase 1}

Bryan S. Sibert, Nathan Fischel-Ghodsian and Jeffrey R. Patton

RNA 2008 14: 1895-1906

References This article cites 52 articles, 25 of which can be accessed free at:

http://rnajournal.cshlp.org/content/14/9/1895.full.html\#ref-list-1

\section{License}

Email Alerting

Receive free email alerts when new articles cite this article - sign up in the box at the Service top right corner of the article or click here.

To subscribe to $R N A$ go to:

http://rnajournal.cshlp.org/subscriptions 